\title{
A Case of Coarctation of the Aorta Diagnosed by Tardus- Parvus Renal Doppler Flow Patterns
}

\author{
Yun-Hwa Jung ${ }^{1}$, Kwan-Wook Kim², Dae-Yoon Kim³, and Chul-Seung Lee ${ }^{3}$
}

Departments of ${ }^{1}$ Internal Medicine, ${ }^{2}$ Family Medicine, and ${ }^{3}$ Radiology, The Armed Force Gangneung Hospital, Gangneung, Korea

A 21-year-old male with secondary hypertension was referred to our facility by a general physician. The patient had no family history or risk factors for hypertension. During the physical examination the patient's blood pressure (BP) measured 190/110 in both arms and no cardiac murmur or bruit was detected. Renal Doppler sonography was performed to identify the potential cause of secondary hypertension, specifically renal artery stenosis. Tardus-parvus pulse waves were noted in both renal intra-arteries (Fig. 1A and 1B); however, a subsequent abdominal computed tomography (CT) did not show any evidence of renal artery stenosis and both adrenal glands looked normal. There was a noted tortuous and engorged superior-epigastric artery along the abdominal wall (Fig. 2).

A chest CT was performed to evaluate supra-diaphrag- matic vessel abnormalities. The CT revealed a stenotic lesion in the isthmus of the aorta (Fig. 3). After making a diagnosis, multiple rib notchings were found on a chest X-ray (Fig. 4). The patient was transferred to another university hospital for surgical treatment.

Coarctation of the aorta (COA) is a congenital malformation, which can cause systemic hypertension and subsequent complications. Because hypertension caused by COA tends to be resistant to medical therapy, early detection and surgical correction are important. Although discrepancies between arm and leg BP and the presence of bruits due to narrowing lesions can aid in the diagnosis of COA, sometimes these signs are not obvious or can be overlooked.

Renal Doppler sonography is a useful and frequently used tool to evaluate secondary hypertension. When
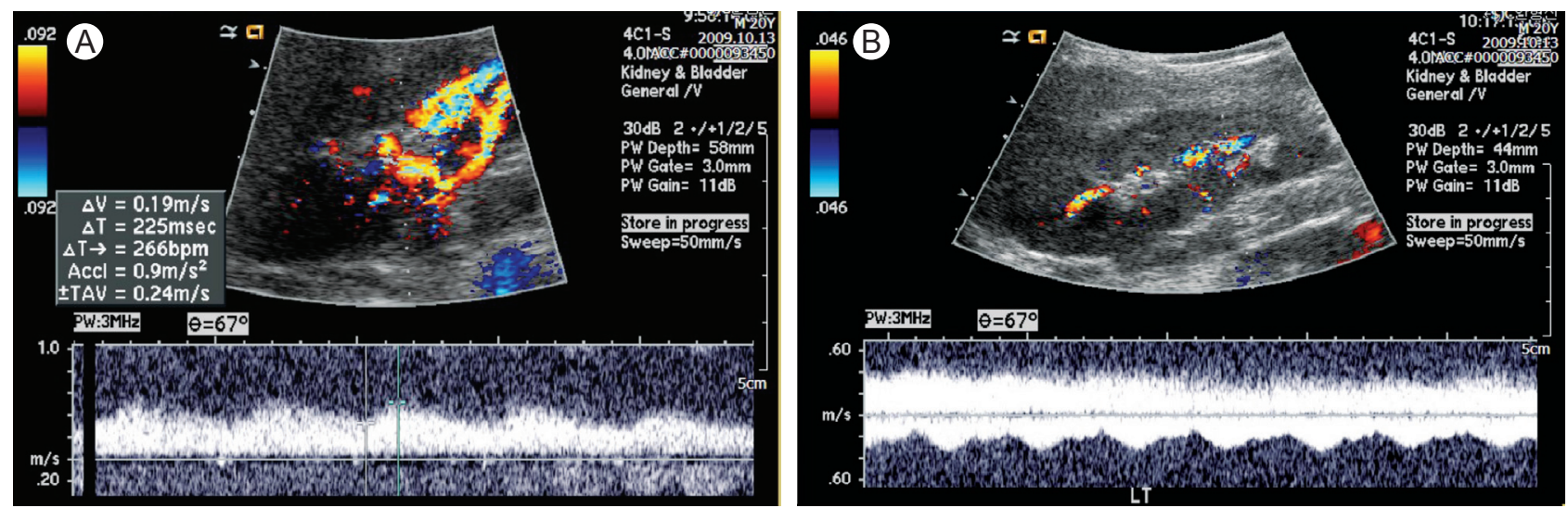

Figure 1. (A) Right renal doppler sono shows tardus-parvus wave pattern. Notice decreased arterial peak velocity and delayed acceleration time which indicates stenosis of proximal artery. (B) Left renal doppler sono shows tardus-parvus wave pattern which is similar to right renal doppler finding. 


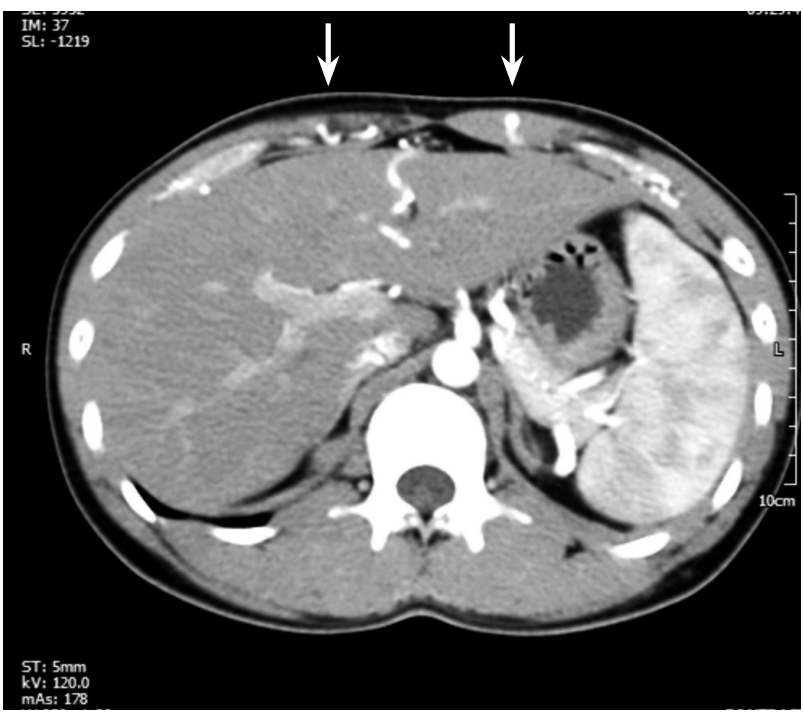

Figure 2. Abdomen contrast computed tomography shows tortous and engorged superior epigastric artery along the abdominal wall (arrows).

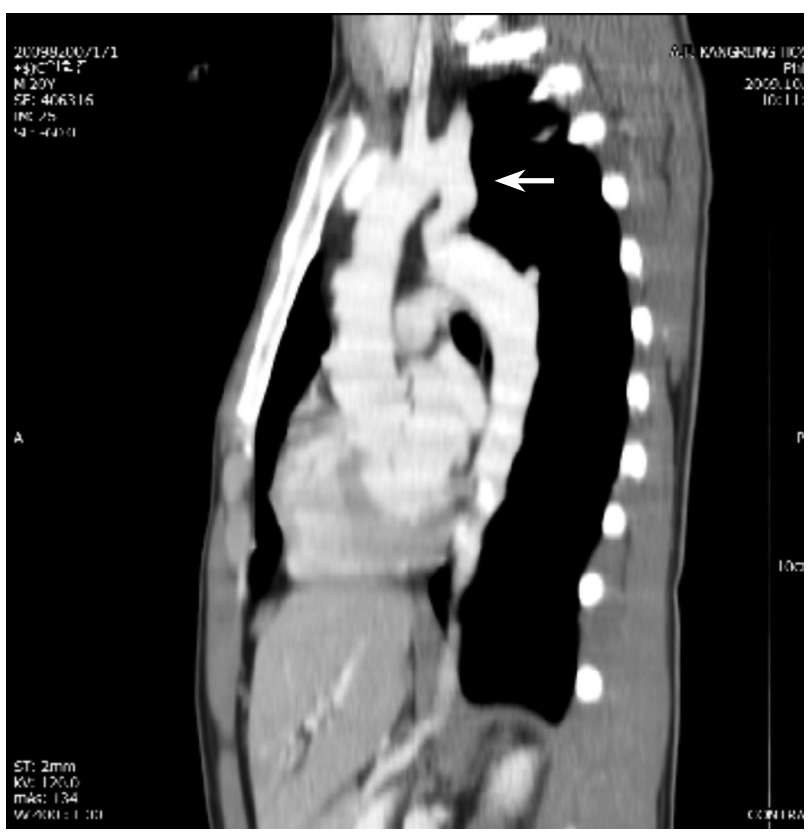

Figure 3. Sagittal contrast enhanced chest computed tomography shows stenosis in the isthmus of aorta (arrow).

bilateral tardus-parvus wave patterns are detected, bilateral renal artery stenosis, aortic stenosis, and COA should be considered. To differentiate between COA and bilateral renal artery stenosis, the pulse wave of the aorta should be checked, which is superior to the renal artery. If

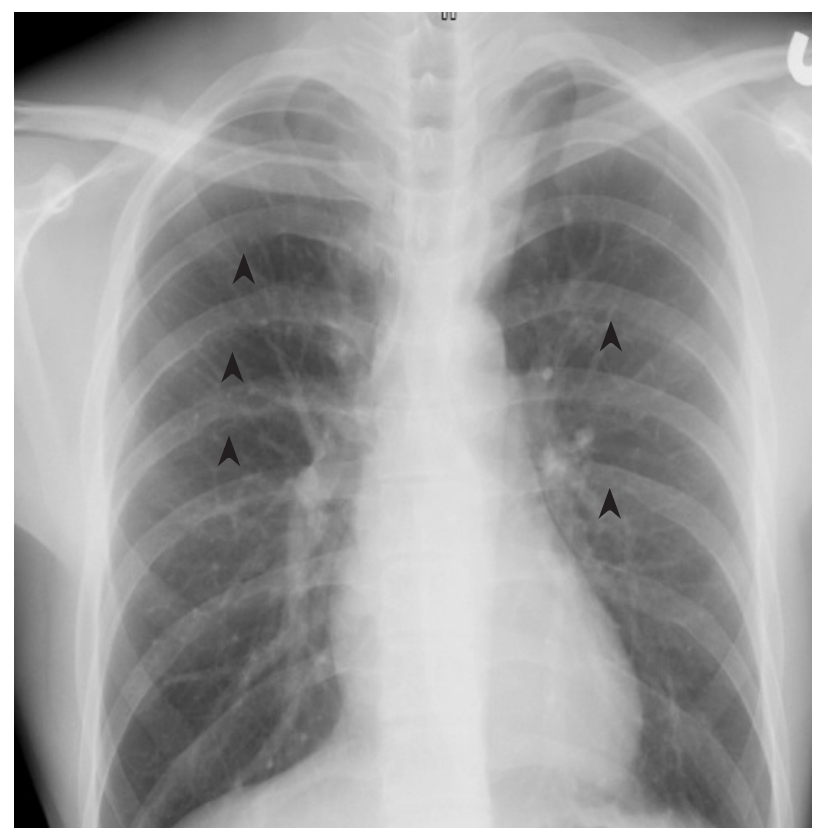

Figure 4. Chest X-ray shows multiple rib notchings (arrowheads).

the aortic pulse wave also shows tardus-parvus patterns, it can either be COA or AS [1]. Additionally, engorged collateral circulations, such as in the internal thoracic artery, intercostal artery, or superior epigastric artery, can help in the diagnosis of COA [2]. (Korean J Intern Med 2011;26:216-217)

Keywords: Coarctation of the aorta; Collateral circulation; Doppler ultrasonography

\section{Conflict of interest}

No potential conflict of interest relevant to this article was reported.

\section{REFERENCES}

1. Tarzamni MK, Nezami N, Ardalan MR, et al. Serendipitous diagnosis of aortic coarctation by bilateral parvus et tardus renal Doppler flow pattern. Cardiovasc Ultrasound 2007;5:44.

2. Leschka S, Alkadhi H, Wildermuth S. Images in cardiology: collateral circulation in aortic coarctation shown by 64 channel multislice computed tomography angiography. Heart 2005;91:1422. 